\section{AB0571 RELATIONSHIP BETWEEN SERUM CALPROTECTIN LEVEL AND PRESENCE OF SUBCLINICAL ATHEROSCLEROSIS AND ARTERIAL STIFFNESS IN PATIENT WITH PSORIATIC ARTHRITIS}

I. T. Cheng ${ }^{1}$, M. LI ${ }^{1}$, E. K. LI ${ }^{1}$, A. P. W. Lee ${ }^{1}$, L. S. Tam ${ }^{1} .{ }^{1}$ The Chinese University of Hong Kong, Medicine and Therapeutics, Hong Kong, Hong Kong (SAR)

Background: Calprotectin is a member of $\mathrm{S} 100$ leukocyte. Serum calprotectin is a sensitive biomarker of disease activity in patients with psoriatic arthritis (PsA). While various $\mathrm{CV}$ risk score only shown modest correlation with augmented CV risk in patient with PsA, whether calprotectin could play an addition role remains uncertain.

Objectives: To elucidate the association between serum calprotectin and subclinical atherosclerosis and arterial stiffness in patient with PsA

Methods: Seventy-eight PsA patient (age: $53 \pm 11$ years, $47(54 \%)$ male) without CV event was recruited into this cross-sectional study. High resolution carotid ultrasound was performed to assess the presence of carotid plaque and intima-media thickness (IMT). Arterial stiffness was measured by branchial-ankle pulse wave velocity (PWV) and augmentation index (Alx). Serum calprotectin level was measured by QUANTA Lite Calprotectin Extended Range ELISA kit from (INOVA Diagnostics, San Diego, CA, USA).

Results: 29/78 (38\%) of patient had carotid plaque (CP+). Subject in $\mathrm{CP}+$ group were of older age and higher inflammatory burden in terms of higher number of swollen joint and longer disease duration. The use of statins were also higher in $\mathrm{CP}+$ group. Serum calprotectin level were significantly higher in CP+ group $(639.2 \mathrm{ng} / \mathrm{ml} \pm 378.2$ in CP- group vs $911.8 \mathrm{ng} / \mathrm{ml} \pm 429.4$ in $\mathrm{CP}+$ group, $p=0.005$ ) (Figure 1). Using multivariate logistic regression analysis, higher level of In calprotectin were significantly associated with presence of carotid plaque (OR: $3.25,95 \% \mathrm{Cl}: 1.22$ to $8.69, p=0.019$ ) after adjusting for baseline covariates. There was also significant correlation between calprotectin level and C-Reactive Protein (CRP) $(r=0.237, p=0.037)$, mean IMT $(\mathrm{r}=0.301, p=0.021)$ and maximum IMT $(r=0.265, p=0.043)$. However, no significant association were observed between calprotectin level and PWV or Alx.

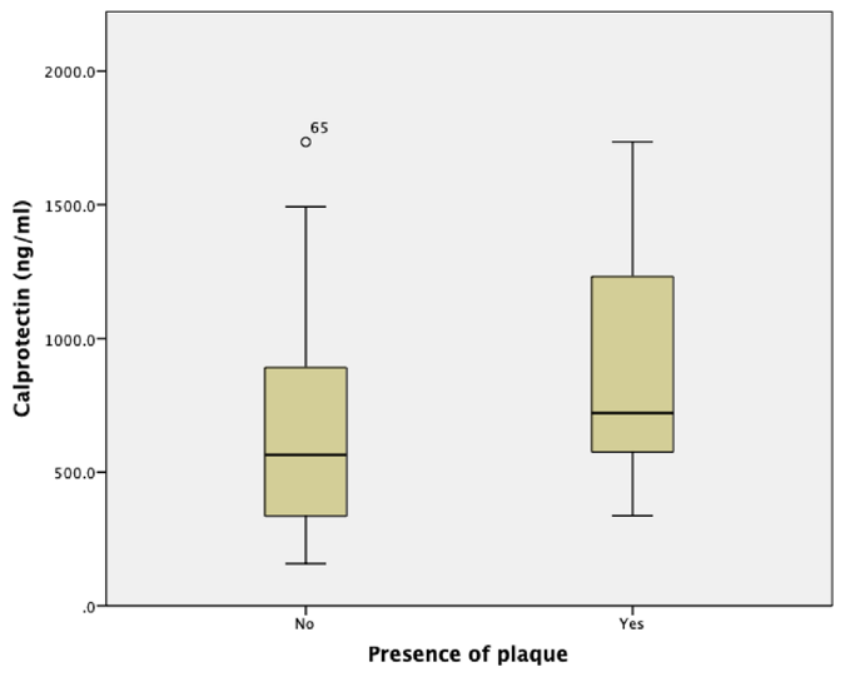

Figure 1.

Conclusion: Increased calprotectin level were associated with presence of plaque and increased IMT. Serum calprotectin may be a novel biomarker for assessing $\mathrm{CV}$ risk in patient with PsA.

Table 1. Multivariate analysis for factors associated with presence of carotid plaque.

\begin{tabular}{llll}
\hline & OR & $95 \% \mathrm{Cl}$ & $p$ \\
\hline Disease duration (years) & 1.10 & 1.02 to 1.19 & 0.018 \\
Ln Calprotectin & 3.25 & 1.22 to 8.69 & 0.019
\end{tabular}

Factors included in the multivariate analysis: age, gender, disease duration, swollen joint count, hyperlipidemia, Framingham 10-year CVD risk $>10 \%$, current use of statins, and calprotectin level

Disclosure of Interests: Isaac T. Cheng: None declared., Martin Li: None declared., Edmund K. Li: None declared., Alex Pui Wai Lee: None declared., LaiShan Tam Grant/research support from: Grants from Norvatis, Pfizer.

DOI: 10.1136/annrheumdis-2021-eular.1249

\section{$\mathrm{AB} 0572$ \\ CARPAL TUNNEL SYNDROME IN PATIENTS WITH PSORIATIC ARTHRITIS; ULTRASONOGRAPHY AND MAGNETIC RESONANCE IMAGING FINDINGS}

E. Akyildiz Tezcan ${ }^{1}$, F. Levendoglu ${ }^{2}$, M. S. Durmaz ${ }^{3}$, H. Kara ${ }^{4}$, E. Balevi Batur ${ }^{2}$, I. Albayrak Gezer ${ }^{2}$, M. K. Korez ${ }^{5} .{ }^{1}$ Cumra State Hospital, Department of Physical Medicine and Rehabilitation, KONYA, Turkey; ${ }^{2}$ Selcuk University Medical Faculty, Department of Physical Medicine and Rehabilitation, KONYA, Turkey; ${ }^{3}$ Selcuk University Medical Faculty, Department of Radiology, KONYA, Turkey; ${ }^{4}$ Erzurum Horosan State Hospital, Department of Physical Medicine and Rehabilitation, ERZURUM, Turkey; ${ }^{5}$ Selcuk University Medical Faculty, Department of Statistics, KONYA, Turkey

Background: Carpal tunnel syndrome(CTS) is the most common form of entrapment neuropathies,caused by compression of the median nerve in the carpa tunnel at the wrist. But there is no gold standard technique for diagnosing CTS. Electrodiagnostic studies (EDS) are generally used but have some limitations. Recent years, magnetic resonance imaging(MRI) and ultrasonography(US) have facilitated the diagnosis of CTS. The median nerve cross section area(CSA) measured by US or MRI has been found to be associated with CTS[1]][2].CTS is usually idiopatic but it can be seen more in some disease. Psoriatic arthritis(PsA) occurs in up to $30 \%$ of people with psoriasis and can have serious debilitating effects on the peripheral joints, spine, tendon insertions, and fingers[3] Because of arthritis, steroid use and flexor tenosynovitis play an important role in the pathogenesis of CTS, we think that CTS can be seen more in PSA patients. Objectives: We aimed to investigate the CTS in PsA patients with EDS, US and $\mathrm{MRI}$ than compare them with healthy controls.

Methods: 68 people, including 39 PsA (according to CASPAR criteria) and 28 healthy volunteers were included in study within 1 year. EDS, US and $\mathrm{MRI}$ were performed within maximum 2 weeks, and measurements were made by different doctors who were blind to other measurments. EDS was started with median and ulnar nerve motor conduction study than continued with sensory conduction studies. CTS diagnose was made according to the routine laboratory standards. The CSA measurement was made from the inner border of the hyperechoic ring around median nerve by using continuous tracing method at psiform bone level. US examinations were performed with a high frequency linear transducer (4-14 MHz), MRI examinations were performed on a 3-T imaging system. The statistical analyses were performed with Statistical Package for the Social Science Program Version 22. Descriptive statistics, T tests, chi-square test, Pearson correlation test were used. Results: No statifically significant difference was found between the groups for demographic characteristics. $12(30.76 \%)$ of 39 PsA patients had CTS whereas CTS was not detected in the control group $(p=0.001)$. US and MRI show larger CSA in PsA patients compared to the healthy control group $(9,49$ $\pm 3,00 \mathrm{~mm}^{2}$ vs $8,30 \pm 1,73 \mathrm{~mm}^{2} \mathrm{p}=0,005,11,24 \pm 3,41 \mathrm{~mm}^{2}$ vs $9,35 \pm 1,81 \mathrm{~mm}^{2}$ $\mathrm{p}<0,001)$; in patients with CTS compared to others $\left(11,63 \mathrm{~mm}^{2} \pm 3,25\right.$ vs $8,60 \pm$ $2,26 \mathrm{~mm}^{2} \mathrm{p}=0,002,13,37 \pm 3,37 \mathrm{~mm}^{2}$ vs $\left.9,90 \pm 1,58 \mathrm{~mm}^{2} \mathrm{p}<0,001\right)$ and in PsA patients which have CTS compared to PsA patients with normal EDS $(11,63 \pm$ $3,25 \mathrm{~mm}^{2}$ vs $8,87 \pm 2,64 \mathrm{~mm}^{2} \mathrm{p}=0,001,13,37 \pm 3,37 \mathrm{~mm}^{2}$ vs $10,52 \pm 3,15 \mathrm{~mm}^{2}$ $\mathrm{p}=0,003)$. When the CSA compared PsA patients which have normal EDS and healthy volunteers; US $(8,87 \pm 2,64$ vs $8,30 \pm 1,73 \mathrm{p}=0,180)$ and MRI $(10,52$ $\pm 3,15$ vs $9,35 \pm 1,81 p=0,026)$ show larger CSA in PsA patients. But differance isn't statistically significant for US measurments. The Pearson correlation coefficient between MRI and US measurements of the CSA was $0.85(P<0,001)$ Conclusion: CTS is more common in patients with PsA. The relationship between CTS diagnosed by EDS and CSA measured by US or MRI was observed in both PsA patients and all participants. Diagnosis can be supported by US or MRI in patients who can not undergo EDS or who do not accept EDS. For PsA patients, cut off values obtained from normal people should not be used. The limitations of our study were that our CTS population was small and most of them was mild. We think that this study will be the precursor of CTS studies in PSA patients. REFERENCES:

[1] M. S. Cartwright et al., "Evidence-based guideline: Neuromuscular ultrasound for the diagnosis of carpal tunnel syndrome," Muscle and Nerve, vol. 46, no. 2, pp. 287-293, Aug. 2012.

[2] M. Ikeda, M. Okada, M. Toyama, T. Uemura, K. Takamatsu, and H. Nakamura, "Comparison of median nerve cross-sectional area on 3-T MRI in patients with carpal tunnel syndrome," Orthopedics, vol. 40, no. 1, pp. e77-e81, Jan. 2017.

[3] C. T. Ritchlin, R. A. Colbert, and D. D. Gladman, "Psoriatic Arthritis," N. Engl. J. Med., vol. 376, no. 10, pp. 957-970, Mar. 2017.

Disclosure of Interests: None declared.

DOI: 10.1136/annrheumdis-2021-eular.1261

\begin{tabular}{l|l}
\hline AB0573 & DUPILUMAB-INDUCED ENTHESITISIARTHRITIS \\
IN PATIENTS WITH ATOPIC DERMATITIS: A \\
RETROSPECTIVE OBSERVATIONAL STUDY
\end{tabular}

J. Nathan ${ }^{1}$, C. Hughes ${ }^{1}$, S. Patel ${ }^{1}$, L. Mathew ${ }^{2}$, C. Smith ${ }^{2}$, A. Pink ${ }^{2}$, R. Woolf ${ }^{2}$, B. Menon ${ }^{1}$, L. B. Kirkham ${ }^{1} .{ }^{1}$ Guys and St Thomas' NHS Foundation Trust, 